\title{
Estudo morfoquantitativo da parede da aorta de ratos wistar idosos treinados com exercício aeróbio
}

\author{
Morphoquantitative study of the aorta's wall in aerobically trained \\ elderly wistar rats
}

M.A. Coura, M.E. Pacheco, H.G. Simões, J.F. Moraes, C.S. Campbell

ARTIGO ORIGINAL | ORIGINAL ARTICLE

\begin{abstract}
RESUMO
O presente estudo teve como objetivo avaliar sob os aspectos morfoquantitativos as mudanças da aorta de Wistar idosos (16 meses) treinados em natação na intensidade do limiar anaeróbio. Dez machos foram divididos em dois grupos, sendo um controle $(n=5)$ e um treinado $(n=5)$, realizaram natação cinco vezes/semana, 30 minutos/dia, durante oito semanas. Para a avaliação aeróbia foi determinado o limiar anaeróbio na primeira, quarta e oitava semanas de treinamento. Após o período experimental, foi realizada a eutanásia e retirada do coração e da aorta, que foram preparados por procedimentos histológicos para colorações com Hematoxilina-Eosina, Weigert e Verhoeff. Foram realizadas avaliações utilizando-se métodos morfométricos e estereológicos. Houve um aumento $(p<.05)$ na espessura, área de secção transversa, fibras elásticas e na distância entre os feixes do núcleo de músculo liso sem influenciar no diâmetro.

Palavras-chave: aterosclerose, inflamação, aorta, exercício físico, hipertrofia da parede do vaso
\end{abstract}

ABSTRACT

The aim of the present study was to evaluate under morphoquantitative aspects possible alterations in the ascending section of the Aorta's endothelium of elderly Wistar rats (16 months of age) trained at an anaerobic threshold intensity. Ten male animals were divided in two experimental groups (control and exercise, each with 5 animals). The exercise group performed swimming five times per week, 30 minutes per day during eight weeks. Anaerobic threshold was evaluated at the first, fourth and eighth week of training. After the intervention the animals were sacrificed and the heart and Aorta were collected. The Aorta was properly fixed and prepared according to the histology procedures for coloring with Hematoxilin-Eosin, Weigert and Verhoeff. Quantitative and qualitative evaluations were performed using morphometric and stereological methods. There was an increase in the thickness, cross sectional area, elastic fibers and distance between the beams and the nucleus of the smooth muscle without alteration of its diameter $(p<.05)$.

Keywords: atherosclerosis, inflammation, aorta, exercise, artery wall hypertrophy

Submetido: 13.05.2012 | Aceite: 18.07.2012

Maritza Alves de Sousa Coura, Herbert Gustavo Simões, Carmen Silvia Grubert Campbell. Universidade Católica de Brasília, Brasília-DF, Brasil.

Mateus Elias Pacheco. Universidade de São Paulo, São Paulo-SP, Brasil.

José Fernando Vila Nova de Moraes. Universidade Federal do Vale do São Francisco, Petrolina-PE, Brasil.

Endereço para correspondência: Maritza Alves de Sousa Coura, Acampamento Rabelo, Rua Dona Alzira de Jesus, casa 6-A, Vila Planalto, Brasília-DF/Brasil, CEP:70834-100.

E-mail:maritzacoura@hotmail.com 
O envelhecimento é um processo fisiológico relacionado com alterações no sistema cardiovascular, sendo considerado um dos fatores de risco para o desenvolvimento de várias doenças como a aterosclerose (Girerd \& Mourad, 2007; Yildiz, 2007). Segundo Yildiz (2007), as alterações cardiovasculares advindas do envelhecimento são as maiores causadoras de mortalidade no mundo. Dentre os mecanismos envolvidos na aterosclerose estão às alterações endoteliais promovidas pelas dislipidemias, hipercoagulação, oferta insuficiente de óxido nítrico $(\mathrm{ON})$, estresse oxidativo, inflamação e disfunção endotelial. Estas alterações na artéria podem ser caracterizadas pelo aumento da espessura $(\delta)$ das túnicas, devido à destruição gradual das fibras elásticas e pelo aumento do depósito de substâncias como o cálcio na matriz extracelular (MEC) (Girerd \& Mourad, 2007).

O sedentarismo está relacionado ao desenvolvimento de doenças (Kannel, 1967) e a prática de exercícios tem a capacidade de prevenir ou retardar o desenvolvimento de placas de ateroma nas artérias (Kramsch, Aspen, Abramowitz, Kremendahl, \& Hood Jr., 1981). O limiar anaeróbio (Lan) é um parâmetro de aptidão aeróbia, sendo definido como a intensidade do exercício a partir da qual ocorre aumento exponencial na concentração de lactato sanguíneo [lac] (Svedahl \& Maclntosh, 2003). Diversos métodos têm sido utilizados para identificar o Lan, como o limiar ventilatório (Wasserman, Whipp, Koyal, \& Beaver, 1973), o limiar glicêmico (Simões, Campbell, Kushnick, Nakamura, Katsanos, \& Baldissera, 2003) e o limiar de lactato (LL) (Svedahl \& Maclntosh, 2003).

A utilização do exercício em intensidade do LL tornou-se vantajosa por determinar a capacidade aeróbia sem que seja necessário atingir o consumo máximo de oxigênio, diminuindo-se assim os riscos cardiovasculares (Wasserman, 2002). A resposta do [lac] ao exercício tem sido considerada válida para diagnóstico da aptidão aeróbia, prescrição e treinamento em humanos
(Svedahl \& Maclntosh, 2003) e ratos (Cunha, Cunha, Segundo, Pacheco, Moreira, \& Simões, 2008).

Gobatto et al. (2001) e Voltarelli, Gobatto, \& Mello (2002) foram os primeiros a publicarem estudos com aplicação de protocolos para determinação do máximo estado estável de lactato (MEEL) e LL em ratos na natação. Sabendo que os princípios gerais que regulam o fluxo de lactato são válidos para ambas as espécies e que é possível identificar o LL em ratos (Cunha et al., 2009), assim como também é possível identificar a MEEL, que acontece por volta de 5.5 mmol.L-1 de lactato nos ratos (Cunha et al., 2009; Gobatto et al., 2001) e nos humanos ocorre por volta de $4 \mathrm{mmol} . \mathrm{L}-1$. Pode-se sugerir que a cinética de lactato seja semelhante entre humanos e ratos, e a utilização destes métodos para avaliação e prescrição de treinamento torna-se muito importante na pesquisa.

Desta forma, o objetivo do presente estudo foi avaliar os efeitos do treinamento de natação na intensidade do limiar anaeróbio sobre os componentes da parede da aorta em ratos Wistar idosos.

\section{MÉTODO}

\section{Amostra}

Foram utilizados 10 ratos Wistar, machos, idosos (478 dias de vida) e sedentários, com peso corporal (PC) médio de $614 \pm 752$ gr, mantidos no Laboratório de Educação Física e Saúde na Universidade Católica de Brasília. Os animais foram alimentados com ração $\left(\right.$ Purina $\left.^{\circledR}\right)$ e água. A temperatura ambiente foi controlada a $25^{\circ} \mathrm{C}$, com fotoperíodo de claro/ escuro de 12 horas. O estudo está de acordo com os princípios éticos do Colégio Brasileiro de Experimentação animal (COBEA) e foi aprovado pela Comissão de Ética em Experimentação animal (CEEA) em 26.08.2008, n. 026, folha 59 do livro 02.

Os ratos foram divididos em 2 grupos ( $p>$ $.05)$, sendo um grupo controle (GC) $n=5$ que não participaram do treinamento, com massa 
corporal (MC) média de $614.0 \pm 83$ gr, e um grupo treinado (GT) $n=5$, submetidos ao treinamento, com MC média de $617 \pm 78$ gr.

\section{Instrumentos e Procedimentos}

A adaptação ao meio líquido consistiu em manter os ratos em contato com a água em tanques à temperatura de $30 \pm 2^{\circ} \mathrm{C}$, durante 2 semanas, 5 vezes por semana, por 30 minutos (Gobatto et al., 2001). Foram realizados testes incrementais na natação para avaliação da capacidade aeróbia dos animais. Os testes consistiram de uma sobrecarga inicial referente ao PC do animal com incrementos de $1 \%$ da MC a cada 3 min até a exaustão. Pausas de $1 \mathrm{~min}$ foram realizadas entre os estágios para troca de cargas e coleta sanguínea (Cunha et al., 2009).

Foi realizado um corte na porção distal da cauda dos animais e amostras sanguíneas de $25 \mu \mathrm{l}$ foram coletadas e colocadas em microtubos contendo $50 \mu \mathrm{l}$ de solução de fluoreto de sódio a $1 \%$. As dosagens de lactato foram feitas pelo método eletroenzimático (Yellow Springs Instruments - USA 2700 - STAT).

A determinação do LL foi realizada a partir do ajuste polinomial da resposta da razão [LAC]/carga durante $\mathrm{o}$ teste incremental (Limiar de Lactato polinomial - LLp) (Cunha et al., 2009).

O protocolo de treinamento foi realizado somente com o GT durante 8 semanas, sendo numa frequência de 5 vezes por semana com duração de 30 minutos cada sessão. A carga do treinamento correspondeu à mesma intensidade do Lan, ou seja, uma sobrecarga de $5 \%$ do PC para cada animal (Gobatto et al., 2001). A eutanásia ocorreu após o treinamento.

Após o sacrifício dos animais coletou-se 5 $\mathrm{mm}$ da aorta. As amostras foram embebidas na solução fixadora de Karnovsky modificada, contendo solução de glutaldeído 3\% (Merck ${ }^{\circledR}$, Alemanha) e formaldeído $1 \%$ (Sigma ${ }^{\circledR}$, USA) em tampão cacodilato de sódio (EMS ${ }^{\circledR}$, BRA \{0.125M; pH 7.4\}) por 72 hs a $4^{\circ} \mathrm{C}$. Em seguida, o material foi submetido à desidratação com passagens sucessivas em soluções de concentrações crescentes de etanóis e diafanizado pelo xilol. A inclusão foi feita em parafina histológica $(56-58)^{\circ} \mathrm{C}$. Foram realizados cortes de $6 \mu \mathrm{m}$ de espessura em um micrótomo (Leica $\mathrm{DMR}^{\circledR}$ ), onde os cortes foram coletados em lâminas para microscopia (Knittel ${ }^{\circledR}$, Alemanha), desparafinizados em estufa a $58^{\circ} \mathrm{C}$ e banhados em séries de xilois; hidratados em soluções de concentrações decrescentes de etanóis e lavados em água destilada.

Foram feitas 3 colorações (Figuras 1A-D): Hematoxilina - Eosina (HE) (Romeis, 1968), para análise morfométrica dos componentes da túnica média da aorta: núcleo de músculo liso, distância entre os feixes de núcleo de músculo liso vascular; Hematoxilina férrica de Verhoeff (Prophet et al., 1992; Verhoeff, 1908) para coloração de fibras elásticas maduras, resorcina de Weigert para coloração de fibras elásticas maduras, resorcina de Weigert pós-oxidação com solução aquosa a $1 \%$ de oxona (Montes, 1996) para coloração de fibras elásticas maduras, eulanínicas e oxitalâmicas. As secções foram desidratadas em soluções de concentrações crescentes de etanóis, diafanizadas em série de xilois, e as lâminas montadas com lamínula e meio de montagem para microscopia (Entellan ${ }^{\circledR}$, Merck $^{\circledR}$, Alemanha).

As imagens da aorta foram capturadas e medidas utilizando-se um microscópio trinocular (Carl Zeiss Microimagin ${ }^{\circledR}$, modelo Axioshop 40, Alemanha) acoplado a uma vídeo-câmera (Axio Cam HRC - ZEISS ${ }^{\circledR}$, Alemanha), conectada a um computador e a um monitor de 17 polegadas. Foram obtidas imagens com um plano focal pequeno. Por meio de um sistema de imagem computadorizado (Software Axion Vision ${ }^{\circledR}$ KS400 Rel. 4.6, Alemanha) foram capturadas imagens das secções histológicas em toda a sua extensão (Brüel, Oxlund, \& Nyengaard, 2005). Os cortes corados com Weigert foram analisados com objetiva de 100x sob imersão, dos quais foram selecionados 10 cortes de cada vaso, de 3 animais de 
cada grupo. Nos cortes, as áreas externa (Ae) e interna (Ai) foram determinados pelo contorno das membranas elásticas externa e interna. Os diâmetros externos (De) foram obtidos pela medida das superfícies da adventícia e o diâmetro interno (Di) desde a superfície do endotélio. As medidas da área da secção transversa (AST) foram obtidas pela diferença entre $\mathrm{Ae}-\mathrm{Ai}(\mathrm{AST}=\mathrm{Ae}-\mathrm{Ai}) ;$ a $\delta$ da túnica média foi obtida dividindo-se por 2 a diferença entre o De - Di $(\delta=$ De - Di/2) e a razão média/luz foi obtida pela razão entre a $\delta$ e o $\mathrm{Di}(\mathrm{R} \mathrm{m} / \mathrm{lz}=\delta /$ Di). De acordo com Mulvany (2002), o conhecimento do Di e $\delta$ da túnica média são parâmetros importantes para indicar a quantidade de material na artéria, fornecendo informações sobre os processos biológicos que determinam a estrutura vascular com relação ao crescimento e/ou regressão.

A quantificação pela técnica da estereologia da túnica média foi realizada com a objetiva de 100X sob imersão no mesmo sistema de aquisição de imagem citado no item anterior, onde foram capturadas imagens das secções histológicas (Brüel et al., 2005). Foi estudado o número (n.), a densidade numérica (QA) de núcleos (Mandarim-de-Lacerda, 1995) e a distância entre os (Figura 1C) núcleos do músculo liso vascular (do centro de um núcleo à outro), bem como densidade de volume (VV) do componente elástico (lamelas e fibrilas) da túnica média da aorta. Segundo Owens e Schwartz (1982), os cálculos de QA de núcleo e VV de lamelas refletem alterações proporcionais da SMC. A análise estereológica em relação às variáveis $\mathrm{n}$. núcleos e densidade numérica de núcleos (QA[nu]), foram feitas médias com 3 animais de cada grupo. Na variável distância entre os feixes do núcleo do músculo liso (Figura 1C), foram analisados
A.
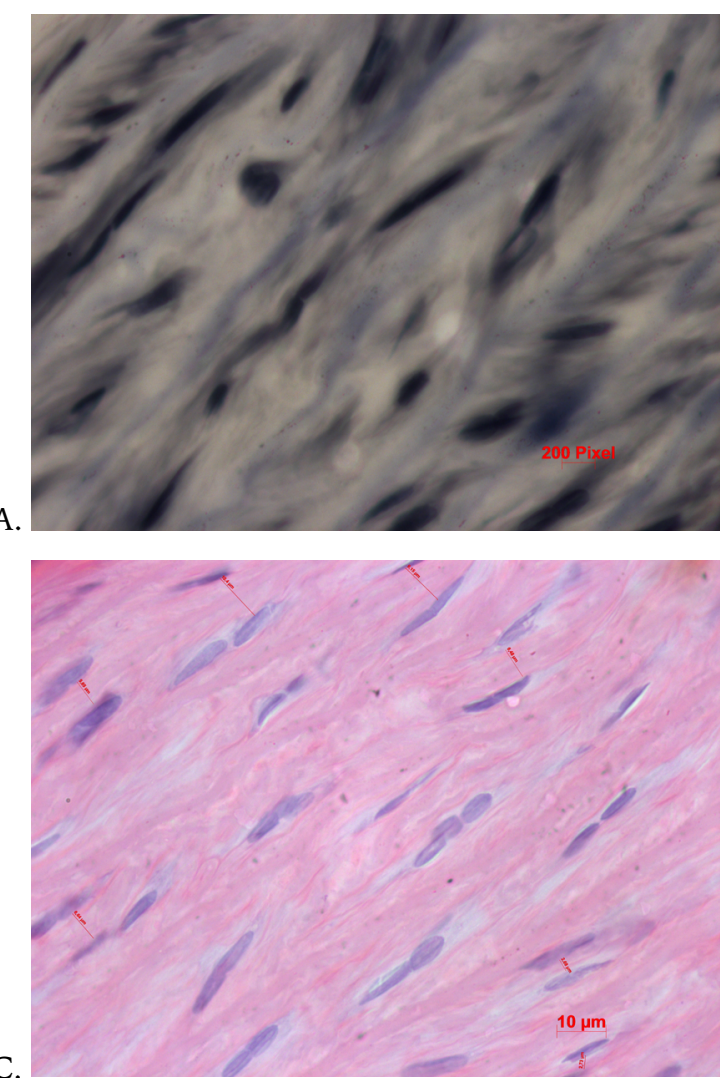

B.

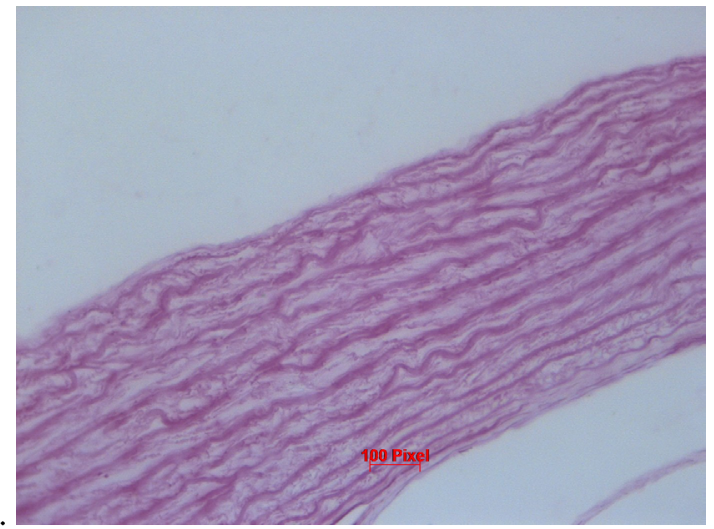

D.

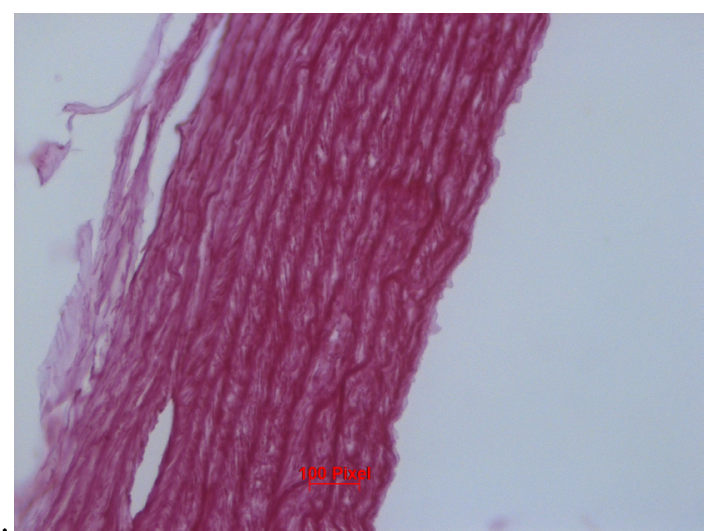

Figura 1. Fotomicrografias da parede da parte ascendente da aorta. A - Fibras elásticas maduras evidenciadas pela técnica de Verhoff; B - Lamelas de fibras elásticas em um vaso de um animal treinado com natação (Weigert); C - Núcleos das células musculares lisas (HE); D - Lamelas em um vaso de rato sedentário 
5 animais por grupo e 100 feixes por animal. $\mathrm{Na}$ variável densidade de volume de lamela VV [lam], a análise estereológica foi feita em 6 campos, aleatórios por animal, 4 animais por grupo. A contagem de pontos das lâminas coradas com HE, utilizando as imagens capturadas ao microscópio com aumento de 100x, foi sobreposta sobre as mesmas em um sistema teste formado por pontos - teste em forma de cruz, equidistante o suficiente para que fossem contados os pontos sobre a estrutura de interesse, obtendo-se o maior grau de acurácia na quantificação (Mandarim-de-Lacerda, 1995; Van Vré et al., 2007), onde os perfis de interesse foram os núcleos de SMC. A contagem de pontos da coloração Verhoeff, onde também foram utilizadas as imagens capturadas do microscópio com aumento de 50x, também foi sobreposto sobre elas um sistema-teste formado por pontos-teste em forma de cruz, sendo a área de contagem delimitada por uma "frame" sem viés, formada por linhas de inclusão e exclusão, com a distância entre os pontos de $1.7 \mathrm{~cm}$, sendo um total de 100 pontos (Brüel et al., 2005), onde o perfil de interesse foram a lamelas elásticas. A contagem de pontos foi realizada através do sistema-teste, contendo 300 pontos, onde a distância entre os pontos foi de $1.7 \mathrm{~cm}$, sendo este sistema-teste colocado sobreposto à imagem na tela do monitor. Os parâmetros estereológicos analisados foram os seguintes: Cálculo da VV da SMC e do componente elástico (lamelas e fibrilas), obtida pela fórmula: $\mathrm{VV}=\mathrm{PP} / \mathrm{PT}(\%)$.Onde: $\mathrm{PP}$ é o número de pontos que tocam a estrutura em estudo; PT é o n. total de pontos do sistema teste multiplicado pelo n. de campos. Para obtenção da Vv, portanto foi realizada a somatória dos valores de todos os campos contados por animal, sendo o resultado desta somatória dividido pelo PT. O Cálculo da QA da SMC obtida pela fórmula: QA $=\mathrm{N} /$ At, sendo o $\mathrm{N}$ o $\mathrm{n}$. de pontos que tocam os núcleos das SMCs dentro da área teste (At) e nas linhas tracejadas. At corresponde à área de $1.7 \mathrm{~cm}$ entre os pontos, onde a frame possuía um total $=600$ pontos. Para obtenção da QA, foi realizada a somatória dos valores de todos os campos contados por animal. Esse valor foi posteriormente dividido por At.

\section{Análise Estatística}

Estatística descritiva com valores de média e desvio padrão ( \pm DP) foi aplicada. Procedimentos para aplicação de função polinomial de segunda ordem foram utilizados para determinação das intensidades relativas ao LLp. Teste-t de Student não pareado foi utilizado para comparações entre grupos. O nível de significância foi $p \leq .05$ (SPSS v.10.0 e Microsoft Excel).

\section{RESULTADOS}

Após a análise do percentual do PC referente ao LLp para os GC e GT verificou-se que as comparações entre os resultados pré e pós treinamento dos grupos não apresentaram diferenças. Quando as comparações foram realizadas entre o GC com GT, verificou-se que o GC apresentou maior LLp que o GT, tanto no momento pré como no pós treinamento $(p<.05)$. Analisando os resultados do PC foi possível notar que treinamento inibiu o aumento do PC dos animais do GT e que o mesmo não ocorreu no GC, pois, os pesos verificados pós-treinamento foram maiores que no pré-treinamento $(p<.05)$ GC. Em relação à variável do peso de $5 \mathrm{~mm}$ da aorta, não apresentaram diferenças entre os grupos pós treinamento (Tabela1).

As áreas e diâmetros da aorta possibilitaram verificar que a Ae, a Ai, o De, o Di e a $\mathrm{R} \mathrm{md/lz,}$ não apresentaram diferenças entre os grupos. Já a AST apresentou-se maior no GT do que no GC $(p<.05)$ (Tabela 1$)$.

Quanto aos resultados do estudo da parede da aorta verificou-se que o GT foi o que apresentou maior $\delta(\mathrm{GC}=102.02 \pm 4.47$; GT $=$ $119.06 \pm 6.29 ; p<.05)$. Entretanto, ao analisar as SMC foi possível notar que o n. de núcleos $(\mathrm{GC}=55.94 \pm 9.24 ; \mathrm{GT}=59.84 \pm 6.83 ; p$ 
Tabela 1

Média e Desvio Padrão das variáveis da área externa (Ae), interna (Ai), diâmetro externo (De), interno (Di), razão média/ luz $(R \mathrm{md} / \mathrm{lz})$, área de secção transversa (AST) e peso da aorta para os grupos controle (GC) e treinado (GT)

\begin{tabular}{ccc}
\hline & GC & GT \\
\hline $\mathrm{Ae}$ & $4.78 \pm 0.74 \mu \mathrm{m}$ & $5.81 \pm 1.06 \mu \mathrm{m}$ \\
$\mathrm{Ai}$ & $3.98 \pm 0.74 \mu \mathrm{m}$ & $4.84 \pm 0.99 \mu \mathrm{m}$ \\
$\mathrm{De}$ & $2618.9 \pm 108.98 \mu \mathrm{m}$ & $2755.73 \pm 210.32 \mu \mathrm{m}$ \\
$\mathrm{Di}$ & $2414.85 \pm 115.82 \mu \mathrm{m}$ & $2517.61 \pm 212.45 \mu \mathrm{m}$ \\
$\mathrm{R} \mathrm{md} / \mathrm{lz}$ & $0.04 \pm 0.00 \mu \mathrm{m}$ & $0.04 \pm 0.00 \mu \mathrm{m}$ \\
$\mathrm{AST}$ & $0.79 \pm 0.01 \mu \mathrm{m}^{2}$ & $0.96 \pm 0.09 * \mu \mathrm{m}^{2}$ \\
Peso Aorta & $5.8 \pm 3.6 \mathrm{mg}$ & $7.5 \pm 2.2 \mathrm{mg}$ \\
\hline
\end{tabular}

${ }^{*} p<.05$ em relação ao GC.

$=.44)$ bem como sua $\mathrm{QA}(\mathrm{GC}=1364.49 \pm$ 166.81; GT $=1459.49 \pm 225.38 ; p=.44)$ não apresentaram diferenças estatísticas entre os GC e GT, permitindo considerar que as modificações de $\delta$ observadas sejam justificadas a partir do aumento do sistema elástico da parede. Estima-se que este desenvolvimento é graças a uma maior Vv de lamelas elásticas em sua parede (figura 2).

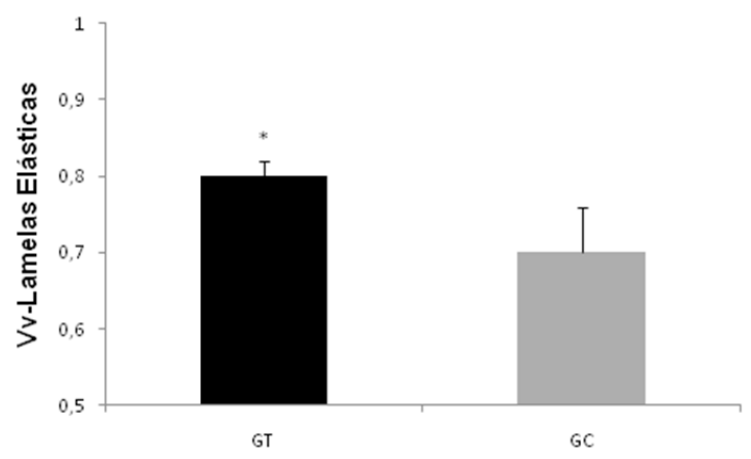

Figura 2. Estudo do sistema elástico da parede da Aorta* $^{*}<.05$ em relação ao grupo controle (GC).

Outro resultado referente às modificações da parede da aorta devido ao protocolo de exercício utilizado são os dados apresentados na figura 3, que contem à distância entre os feixes de músculo liso na parede arterial e sua distribuição para os GC e GT. A aorta do GS apresentou mais de $90 \%$ dos feixes com distância entre $2 \mu \mathrm{m}$ e $4 \mu \mathrm{m}$, sendo que feixes com distância de $2 \mu \mathrm{m}$ são os mais frequentes (56\%). O GT apresentou 7\% dos feixes com distância de $2 \mu \mathrm{m}$. Ainda, observe que maior parte dos feixes musculares lisos possui distância de
$6 \mu \mathrm{m}(22 \%)$ e que mais de $60 \%$ dos feixes apresentaram distância de $4 \mu \mathrm{m}$ a $10 \mu \mathrm{m}$. Por fim, enquanto o GS nem apresentou feixes com distância maior que $8 \mu \mathrm{m}$, no GT verificaram-se feixes com até $22 \mu \mathrm{m}$ de distância.

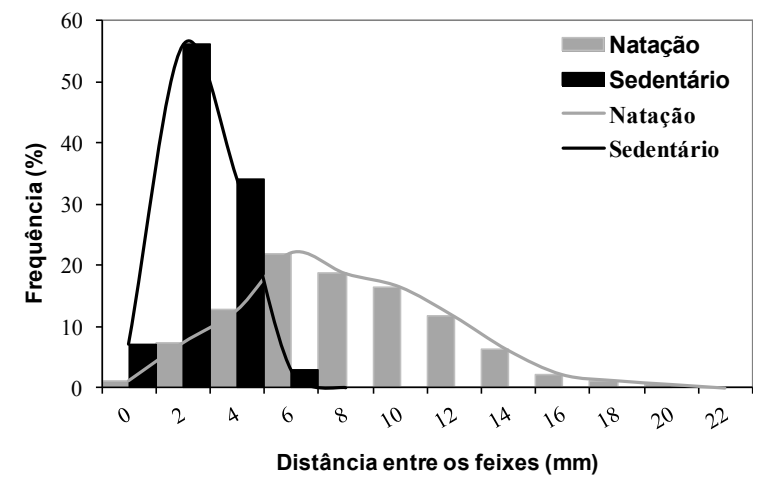

Figura 3. Histograma da distância entre os feixes de músculo liso para os grupos controle e treinado

\section{DISCUSSÃO E CONCLUSÕES}

O estudo avaliou sob os aspectos morfoquantitativos as mudanças da aorta de Wistar idosos treinados em natação na intensidade do limiar anaeróbio. $\mathrm{O}$ treinamento promoveu um aumento $(p<.05)$ na espessura, área de secção transversa, fibras elásticas e na distância entre os feixes do núcleo de músculo liso sem influenciar no diâmetro da aorta. Horta et al. (2005), verificaram os efeitos do treinamento em esteira, $1 \mathrm{~h} /$ dia, 5 dias/semana, durante 20 semanas, sobre a remodelação da aorta em ratos normotensos e SHR. Os autores analisaram a $\delta$ total da parede da aorta e da túnica média e verificaram o $\mathrm{n}$. de lamelas e constataram que o exercício foi eficaz para manter a 
estrutura da aorta dos SHR em condições similares a dos normotensos. Em relação ao Di da aorta, os dados demonstram que no GT, o exercício não promoveu um aumento em relação ao GC. Este dado corrobora com os resultados de Segal, Kurjiaka, e Caston (1993), que treinaram ratos Sprague-Dawley, durante 16 semanas em corrida, 7 vezes por semana, 3 hs/dia, a uma intensidade de $65-80 \%$ do $\mathrm{VO}_{2}$ máx., onde também não observou diferença entre os GT e GC. Esses dados vão contra os resultados de outros autores, onde ocorreu uma diminuição da luz das arteríolas durante o desenvolvimento da hipertensão (Briones et al., 2006; Folkow, 1982).

Stevenson, Feleki, Rechnitzer, e Beaton (1964), demonstraram um aumento do diâmetro de artéria de ratos exercitados, onde foram associados ao aumento do coração e uma mudança adaptativa devido à alterações hemodinâmicas. No que se refere à $\mathrm{Rmd} / \mathrm{lz}$ das aortas dos animais utilizados no estudo, não foram observadas diferenças entre os grupos. Segundo Mulvany (2002), quando há um aumento dessa razão pode ser devido à hipertrofia, hiperplasia e remodelagem da musculatura lisa vascular. A ação do exercício em reduzir essa razão é importante, porque está relacionada com a resistência do vaso. Quanto maior a Rmd/luz, maior é a resistência do vaso ao fluxo sanguíneo e consequentemente maior será a pressão. Ainda de acordo com o mesmo autor, artérias de resistência apresentam um processo de remodelamento eutrófico interno, sem alteração na composição dos materiais constituintes. Entretanto, em decorrência das diferenças estruturais encontradas em artérias, o processo de remodelamento difere (Bund, 2001). Em relação à $\delta$ da parede da aorta, ocorreu um aumento no GT, quando comparado com o GC, corroborando com os achados de Segal et al. (1993). Sendo que, quando analisamos o n. e a densidade de núcleos de SMC e a distância entre os feixes, concordamos que foi devido à hipertrofia da SMC ou um aumento da MEC em resposta ao aumento do estresse da parede do vaso durante o exercício. Ao contrário dos resultados de Horta, Carvalho, e Mandarim-de-Lacerda (2005), onde um grupo SHR foi submetido ao exercício $1 \mathrm{~h} /$ dia, 5 dias/ semana, durante 20 semanas. Quando comparados os grupos Wistar sedentários e SHR-EX com o grupo SHR-SED, apresentaram menor $\delta$ artérial e maior QA de núcleos. Matsuda et al. (1989), concluíram que a natação realizada por ratos idosos, treinados $1 \mathrm{~h} / \mathrm{dia}, 6$ dias/ semana, durante 16 semanas resultou em uma aorta mais forte e extensível, o que está relacionado com a maior quantidade de elastina e com menos alterações degenerativas. Uma das limitações do estudo, foi devido a perda do número da amostra no micrótomo na hora de dissecar a aorta, futuros estudos necessitam ser realizados para observar se o diâmetro da aorta ascendente sobre alterações com uma amostra aumentada. Em relação à Vv de lamelas elásticas e os resultados acima, concluímos que a aorta ascendente de wistar idosos, treinados em natação no LL durante 8 semanas, apresentaram um aumento da $\delta$ sem alterar o Di. Essa adaptação deveu-se à hipertrofia de SMC ou um aumento das lamelas elásticas ocasionando uma artéria mais forte e conseqüentemente reduzindo o estresse tangencial na artéria durante o exercício e o repouso.

\section{Agradecimentos:}

Nada declarado.

\section{Conflito de Interesses:}

Nada declarado.

\section{Financiamento:}

Nada declarado.

\section{REFERÊNCIAS}

Briones, A. M., Xavier, F. E, Arribas, S. M., González, M. C., Rossoni, L. V.,...Alonso, M. J. (2006). Alterations in structure and mechanics of resis- 
tance arteries from ouabain-induced hypertensive rats. American Journal of Physiology - Heart and Circulatory Physiology, 291 (1), H193-201.

Bruel, A., Oxlund, H., \& Nyengaard, J. R. (2005). The total length of myocytes and capillaries, and total number of myocyte nuclei in the rat heart are time-dependently increased by growth hormone. Growth Hormone \& IGF Research, 15(4), 256-264.

Bund, S. J. (2001). Spontaneously hypertensive rat resistance artery structure related to myogenic and mechanical properties. Clinical Science. (Lond), 101(4), 385-393.

Cunha, R. R., Cunha, V. N. R., Segundo, P. R., Moreira, S. R., Kokubun, E., Campbell, C. S. G.,...Simões, H. G. (2009). Determination of the lactate threshold and maximal blood lactate steady state intensity in aged rats. Cell Biochemistry and Function, 27(6), 351-357.

Cunha, V. N. C., Cunha, R. R., Segundo, P. R., Pacheco, M. E., Moreira, S. R., \& Simões, H. G. (2008). Oito semanas de treinamento moderado não altera a carga correspondente ao limiar de lactato em ratos idosos. Revista Portuguesa de Ciências do Desporto, 8(2), 277-283.

Folkow, B. (1982). Physiological aspects of primary hypertension. Physiological Reviews, 62(2), 347-504.

Girerd, X., Mourad, J. J., Boutouvrie, P., Benetos, A., Laurent, S., \& Safar, M. (2007) Effects of aging on arterial function in man. Minerva Cardioangio, 5, 497-502.

Gobatto, C. A., Mello, M. A. R., Sibuya, C. Y., Azevedo, J. R. M., Santos, L. A., \& Kokubun, E. (2001). Maximal lactate steady state in rats submitted to swimming exercise. Comparative Biochemistry and Physiology part A: Molecular and Integrative Physiology, 130(1), 21-27.

Horta, P. P., Carvalho, J. J., \& Mandarim-de-Lacerda, C. A. (2005). Exercise training attenuates blood pressure elevation and adverse remodeling in the aorta of spontaneously hypertensive rats. Life Sciences, 77(26), 3336-3343.

Kannel, W. B. (1967). Habitual level of physical activity and risk of coronary heart disease: the
Framingham study. Canadian Medical Association Journal, 96(12), 811-812.

Kramsch, D. M., Aspen, A. J., Abramowitz, B. M., Kremendahl, T., \& Hood Jr., W. B. (1981). Reduction of coronary atherosclerosis by moderate conditioning exercise in monkeys on an atherogenic diet. The New England Journal of Medicine, 305(25), 1483-1489.

Mandarim-de-Lacerda, C. A. (1995). Métodos quantitativos em morfologia. $1^{\text {a }}$ ed. Rio de Janeiro: Eduerj.

Matsuda, M., Nosaka, T., Sato, M., Iijima, J., Ohshima, N., \& Fukushima, H. (1989). Effects of exercise training on biochemical and biomechanical properties of rat aorta. Angiology, 40(1), 51.

Montes, G. (1996). Structural biology of the fibres of the collagenous and elastic systems. Cell Biology International, 20(1), 15-27.

Mulvany, M. (2002). Small artery remodeling and significance in the development of hypertension. News in physiological sciences: an international journal of physiology produced jointly by the International Union of Physiological Sciences and the American Physiological Society, 17, 105.

Mulvany, M. J., Baumbach, G. L., Aalkjaer, C., Heagerty, A. M., Korsgaard, N.,...Schiffrin, E. L., (1996) Vascular remodeling. Hypertension, 28(3), 505-506.

Owens, G. K., \& Schwartz, S. M. (1982). Alterations in vascular smooth muscle mass in the spontaneously hypertensive rat. Role of cellular hypertrophy, hyperploidy, and hyperplasia. Circulation Research, 51(3), 280-289.

Prophet, E. B, Mills, B., Arrington, J. B., Sobin, L. H. (Eds.). (1992). Armed Forces Institute of Pathology. Laboratory methods in histotechnology. Washington, DC: American Registry of Pathology.

Romeis, B. (1968). Mikroskopische technik. München, Wien: R. Ouldenbourg Verlag.

Segal, S. S., Kurjiaka, D. T., \& Caston A. L. (1993). Endurance training increases arterial wall thickness in rats. Journal of Applied Physiology, 74(2), 722-726 
Simões, H. G., Campbell, C. S. G., Kushnick, M. R., Nakamura, A., Katsanos C. S., Baldissera, V. (2003). Blood glucose threshold and the metabolic responses to incremental exercise tests with and without prior lactic acidosis induction. European Journal of Applied Physiology, 89 (6), 603-611.

Stevenson, J. A., Feleki, V., Rechnitzer, P., Beaton, J. R. (1964). Effect of Exercise on Coronary Tree Size in the Rat. Circulation Research, 15, 265-269.

Svedahl, K. \& MacIntosh, B. R. (2003). Anaerobic threshold: the concept and methods of measurement. Canadian Journal of Applied Physiology, 28(2), 299-323.

Van Vré, E., van Beusekom, H. M., Vrints, C. J., Bosmans, J. M., Bult, H., \& Van der Giessen, W. (2007). Stereology: a simplified and more time-efficient method than planimetry for the quantitative analysis of vascular structures in different models of intimal thickening. Cardiovascular Pathology, 16(1), 43-50.
Verhoeff, F. (1908). Some new staining methods of wide applicability. including a rapid differential stain for elastic tissue. Journal of the American Medical Association (JAMA), 50(11), 876.

Voltarelli, F., Gobatto, C., \& Mello, M. (2002). Determination of anaerobic threshold in rats using the lactate minimum test. Brazilian Journal of Medical and Biological Research, 35, 1389-1394.

Wasserman, K. (2002). Anaerobic threshold and cardiovascular function. Monaldi Archives of Chest Disease, 58(1), 1-5.

Wasserman, K., Whipp, B. J., Koyal, S. N., \& Beaver, W. L. (1973). Anaerobic threshold and respiratory gas exchange during exercise. Journal of Applied Physiology, 35(2), 236-243.

Yildiz, O. (2007). Vascular smooth muscle and endothelial functions in aging. Annals of the New York Academy of Science, 1100, 353-360.

(cc) EY-NC Todo o conteúdo da revista Motricidade está licenciado sob a Creative Commons, exceto quando especificado em contrário e nos conteúdos retirados de outras fontes bibliográficas. 